\title{
Effects of VEGF suppression by small hairpin RNA interference combined with radiotherapy on the growth of cervical cancer
}

\author{
L. Qi ${ }^{1}$, L.N. Xing ${ }^{1}$, X. Wei ${ }^{2}$ and S.G. Song ${ }^{1}$ \\ ${ }^{1}$ Department of Cancer Radiotherapy, \\ The 2nd Affiliated Hospital of Harbin Medical University, \\ Harbin, Heilongjiang, China \\ ${ }^{2}$ Department of Nephrology, Space Center Hospital, Beijing, China \\ Corresponding author: L.N. Xing \\ E-mail: linaxingcn@163.com
}

Genet. Mol. Res. 13 (3): 5094-5106 (2014)

Received May 16, 2013

Accepted October 2, 2013

Published July 7, 2014

DOI http://dx.doi.org/10.4238/2014.July.7.2

\begin{abstract}
This study aimed to investigate the inhibition of vascular endothelial growth factor (VEGF) by small hairpin RNA (shRNA) interference combined with radiotherapy on the growth of cervical cancer SiHa cell xenografts in nude mice. The effective pVEGFshRNA plasmid was screened by reverse transcription-polymerase chain reaction (RT-PCR), and the cell apoptosis rate was estimated by flow cytometry. A nude mouse cervical xenograft model was established and all models were divided into four groups: blank control, VEGF shRNA, radiotherapy, and combined treatment. We calculated the tumor growth curve and the inhibitory rate. The histopathological changes of the microvascular density and VEGF protein expression were observed by immunohistochemistry. The expressions of VEGF and hypoxia inducible factor- $1 \alpha(\mathrm{HIF}-1 \alpha)$ proteins in the tumor tissue were analyzed via Western blot. VEGF shRNA interference inhibited the expression of radiation-induced VEGF $(\mathrm{P}<0.05)$, induced apoptosis ( $\mathrm{P}$ $<0.05)$, downregulated the HIF-1 $\alpha$ protein, and reduced angiogenesis.
\end{abstract}


Compared with the other three groups, the combined treatment group showed the most significant effects $(\mathrm{P}<0.01)$. VEGF shRNA interference combined with radiotherapy promotes the radiosensitivity of tumors via improvement of the hypoxic microenvironment.

Key words: Cervical cancer; Vascular endothelial growth factor; SiHa cells; RNA interference; Radiosensitivity

\section{INTRODUCTION}

Cervical cancer is one of the most common female malignancies (Parkin et al., 1999; Kamangar et al., 2006), and is the leading cause of female reproductive system morbidity. In recent years, the incidence of cervical cancer has increased and manifested in the younger population. Moreover, a number of patients have been diagnosed in the IIB phase, and concurrent chemoradiotherapy has become the standard therapeutic regimen for medium-term and advanced cervical cancer. However, in clinical practice, we have found a considerable number of patients suffering from radiation insensitivity. Despite continuous technological innovations on radiotherapy equipment, its effect remains unsatisfactory, especially with respect to its negative effects of local recurrence and distant metastasis.

Recently, molecular targeted therapy has received a great deal of attention in the treatment of cancer, and in-depth studies have been conducted for anti-angiogenic targeted therapy. Vascular endothelial growth factor (VEGF), a specific growth factor associated with the angiogenesis, occurrence, development, invasion, and metastasis of tumors, is expressed in most solid tumors. Several studies have regarded the VEGF family and its corresponding receptors as anti-angiogenic target molecules, such as Avastin (VEGF-A monoclonal antibody) and Macugen (VEGF-165 antisense nucleotide), both of which have been approved by the Food and Drug Administration (FDA) (Miller et al., 2007; McDermott and George, 2010). In early clinical trials, treatment with individual anti-angiogenic therapy molecules exhibited weak anti-tumor effects; however, combined with chemotherapy, the limitations of each treatment were overcome (O'Reilly, 2006). Thus, the combined treatment method has become one of the hot spots of cancer research. Some studies have confirmed that the expression of VEGF increases in a stepwise manner in normal tissue (Cervical Intraepithelial Neoplasia Organization) and cervical cancer tissue, and shows a higher expression in the late stage of cervical cancer. Furthermore, radiation can induce increased expression of VEGF; thus, anti-VEGF therapy may have an important role in improving the efficacy of radiotherapy. Few studies have been conducted to determine whether anti-VEGF therapy might improve the radiosensitivity of tumors. The current study used RNA interference (RNAi) technology to lower the expression of VEGF in SiHa cells, and further evaluated its impact on tumor cell apoptosis and sensitivity to radiation in nude mice.

\section{MATERIAL AND METHODS}

\section{Plasmid construction}

Based on the Genbank VEGF mRNA sequence, two small hairpin RNA (shRNA) plas- 
mid sequences (pVEGF-shRNA) targeting different coding regions of the human VEGF165 mRNA and a negative control plasmid sequence (pNeg-shRNA) were designed. These plasmids contained a U6 promoter-driven green fluorescent protein (GFP), which can be tracked by fluorescence microscopy to estimate the transfection efficiency (Jima, Shanghai, China). The shRNA sequences are shown in Table 1.

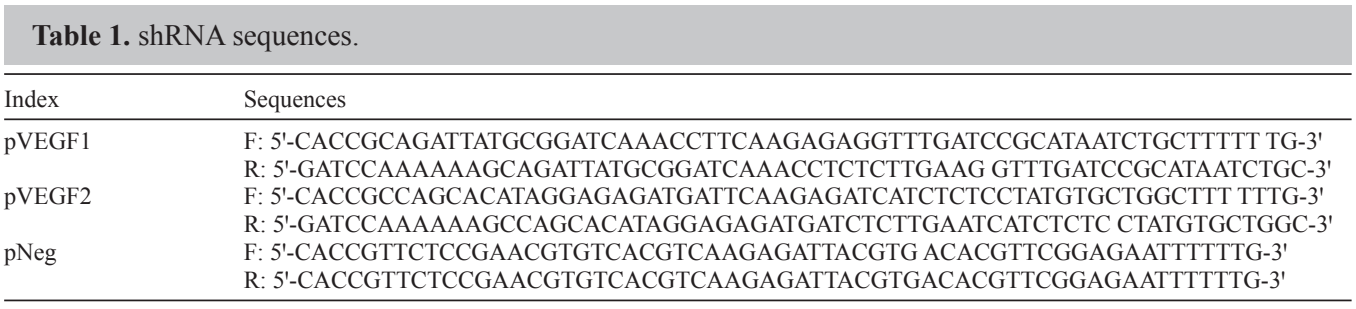

\section{Cell culture and transfection}

SiHa cells (Chinese Academy of Sciences, Shanghai Science and Technology Center of Biological Cells) were grown in Dulbecco's modified Eagle's medium (DMEM) supplemented with $10 \%$ fetal bovine serum (FBS) and antibiotics at $37^{\circ} \mathrm{C}$ and $5 \% \mathrm{CO}_{2}$ The cells in logarithmic growth phase were used for our experiments. A total of $1.5 \times 10^{5} \mathrm{Ge} / \mathrm{mL}$ SiHa cells were seeded in 6 -well plates, and then $2 \mathrm{~mL}$ culture medium without antibiotics was added to each well. The samples were dived into four groups: pVEGF1, pVEGF2, negative plasmid pNeg, and untransfected. Cells were grown to $60 \%$ confluence, and were then transfected in OptiMEM with $10 \mu \mathrm{L}$ Lipofectamine (Invitrogen; USA) and $4 \mu \mathrm{g}$ shRNA. The shRNA and Lipofectamine were diluted in the OptiMEM medium for at least $5 \mathrm{~min}$, and then mixed. After $20 \mathrm{~min}$, the mixtures were added to the 6-well plates, and the optiMEM was changed to DMEM with $10 \%$ FBS after $6 \mathrm{~h}$. The transfection efficiency was observed with a fluorescence microscope and calculated as: transfection efficiency $(\%)=$ the number of green fluorescent cells/total cells x $100 \%$.

\section{Screening of effective pVEGF-shRNA sequences}

Transfected cells were harvested after $48 \mathrm{~h}$. After the extraction of total cellular RNA and the detection of their integrity, purity, and concentration, cDNA was synthesized using the Trizol reverse transcription (RT) kit (Promega; USA). The primer sequences and polymerase chain reaction (PCR) conditions are shown in Table 2. The primer sequences for VEGF were: forward, 5'-TTGCCTTGCTGCTCTACCTC-3' and reverse, 5'-TGCATGGTGATGTTGGACTC-3'. The $\beta$-actin gene was used as the internal reference with the following primer sequences: forward, 5'-GGCACCCAGCACAATGAA-3' and reverse, 5'-GGAAGGTGGACAGCGAGG-3'. VEGF and $\beta$-actin RT-PCR primer sequences were synthesized by the Shanghai Biological Engineering Company.

Approximately $5 \mu \mathrm{L}$ PCR product was electrophoresed on $2 \%$ agarose gel. The results were analyzed with a Tanon GIS 2010 gel imaging analyzer. An absorbance ratio of VEGF/ $\beta$ actin was obtained. 
Table 2. Target gene primer sequences, PCR condition and amplified product.

\begin{tabular}{|c|c|c|c|c|}
\hline Target gene & Primer sequences & Product (bp) & $\mathrm{T}\left({ }^{\circ} \mathrm{C}\right)$ & Cycles \\
\hline VEGF & $\begin{array}{l}\text { Forward 5'-TTGCCTTGCTGCTCTACCTC-3' } \\
\text { Reverse 5'-TGCATGGTGATGTTGGACTC-3' }\end{array}$ & 280 & 53 & 35 \\
\hline$\beta$-actin & $\begin{array}{l}\text { Forward 5'-GGCACCCAGCACAATGAA-3' } \\
\text { Reverse 5'-GGAAGGTGGACAGCGAGG-3' }\end{array}$ & 98 & 53 & 35 \\
\hline
\end{tabular}

$\mathrm{T}\left({ }^{\circ} \mathrm{C}\right)=$ Annealing temperature.

\section{Cell apoptosis}

Transfected cells were harvested after $48 \mathrm{~h}$, fixed with $70 \%$ cold ethanol at $4{ }^{\circ} \mathrm{C}$ overnight, and then treated with a staining solution containing RNase and propidium iodide (PI) in the dark at room temperature for $30 \mathrm{~min}$. Flow cytometry and the cell cycle software was used to estimate the percentage of apoptosis (Flow cytometry cycle detection kit, FACSort flow cytometry, B \& D companies; USA).

\section{Establishment of a tumor-burdened nude mouse model}

Forty 6- to 8-week-old female BALC/C nude mice, weighing 20 to $25 \mathrm{~g}$ (Animal Center of Beijing; China), were reared in a specific non-antigen environment. Each nude mouse received subcutaneous inoculation of $0.2 \mathrm{~mL}\left(5 \times 10^{6}\right.$ cells $\left./ \mathrm{mL}\right)$ SiHa single cell suspension in the thigh lateral region. After 2 weeks, a tumor mass reaching $6 \mathrm{~mm}$ in diameter was judged as a successful model. The 40 nude mice were all up to the standard and were randomly divided into four groups $(\mathrm{N}=10)$ to receive respective peritumoral subcutaneous injections: blank control group [0.1 mL phosphate-buffered saline (PBS) from day 1 to day 5], VEGF shRNA interference group $(0.1 \mathrm{~mL}$ complex solution containing $50 \mu \mathrm{g}$ VEGF shRNA and $125 \mu \mathrm{L}$ liposome from day 1 to day 5), radiotherapy group (after the last treatment to the blank control group, $6 \mathrm{MV}-\mathrm{X}$ line accelerator for $6 \mathrm{~h}, 100 \mathrm{~cm}$ source skin distance, 5 x $5 \mathrm{~cm}$ irradiation field, 10 Gy total irradiation dose, disposable), and the combined treatment group (after the last treatment to the VEGF shRNA interference group, radiotherapy was performed as in the radiotherapy group). This study was carried out in strict accordance with the recommendations in the Guide for the Care and Use of Laboratory Animals of the National Institutes of Health. The animal use protocol was reviewed and approved by the Institutional Animal Care and Use Committee (IACUC) of Harbin Medical University.

\section{Tumor growth curve}

The maximum diameter (a) and minimum diameter (b) of the tumor were measured with a Vernier caliper every three days, and at the same time, the behavior, diet, and weight of nude mice were observed. After removal of the xenografts of nude mice, the tumor volume (V) was calculated as: $\mathrm{V}=\mathrm{ab}^{2} / 2$. The tumor growth curve and the tumor growth inhibition rate (TIR) were determined according to the average $\mathrm{V}$ in each group: TIR $=$ (control group $\mathrm{V}$ - treatment group V) / control group V x 100\%.

\section{Immunohistochemistry}

Transplanted tumor tissues were cut into $3 \mathrm{~mm}$ sheets, fixed into $40 \mathrm{~g} / \mathrm{L}$ form- 
aldehyde for $24 \mathrm{~h}$, embedded in paraffin, and sliced into 5- $\mu \mathrm{m}$ sections. Sections were then dewaxed and hydrated, and endogenous enzymes were removed with $3 \%$ hydrogen peroxide, antigens were retrieved under high temperature and high pressure, and incubated with anti-human VEGF antibody. After diaminobenzadine (DAB) staining, the hematoxylin and gum histopathological changes and the expression of the VEGF protein were observed under a microscope. The VEGF expression was apparent in the cytoplasm and was brownish-yellow. According to the percentage of positive staining cells in each group of slices, the cells were divided into: negative $(-)(<10 \%)$, weakly positive $(+)(11$ to $30 \%)$, positive $(++)(31$ to $50 \%)$, and strongly positive $(+++)(>$ $50 \%)$.

\section{Measurement of microvessel density (MVD)}

The MVD calculation was based on the Weidner method. First, three microvascular intensive areas in the whole paraffin sections under low magnification (100X) were identified in each slice; the counter was used to estimate the numbers under a high power field (400X). Five fields of each slice were counted, three sections were counted in each group, and the average value obtained represented the MVD.

\section{Quantitative analysis of the VEGF tissue and hypoxia inducible factor 1- $\alpha \alpha(\mathrm{HIF}-1 \alpha \alpha)$ protein}

We fully ground and cracked every $100 \mathrm{mg}$ transplanted tumor tissue with 1 $\mathrm{mL}$ tissue lysate. After centrifugation, the protein in the supernatant was obtained; all procedures were conducted on ice. The concentration was measured using the bicinchoninic acid (BCA) method. One hundred micrograms sample protein from each sample was electrophoretically separated by $10 \%$ sodium dodecyl sulfate-polyacrylamide gel electrophoresis (SDS-PAGE). VEGF and HIF-1 $\alpha \alpha$ proteins were transferred to a polyvinylidene fluoride (PVDF) membrane through electrical transfer film with a $1 \mathrm{~mA} /$ $\mathrm{cm}^{2}$ constant current. Membranes were incubated with goat anti-human monoclonal antibody VEGF $(1: 100)$ and HIF-1 $\alpha(1: 1000)$ diluted in 5\% skim milk-Tris-buffered saline with Tween (TBST) at $4^{\circ} \mathrm{C}$ overnight. On the second day, the membranes were washed three times with TBST for 10 min each and incubated with alkaline phosphatase-labeled mouse anti-goat secondary antibody $(1: 1000)$ (Beijing Zhongshan Golden Bridge Biotechnology Co., Ltd; China) at room temperature for $2 \mathrm{~h}$. After the washing phase, banding development was conducted using the alkaline phosphatase chromogenic reagent in the dark. The Tanon GIS 2010 image analysis system was used to analyze the results. The absorbance ratio of VEGF and HIF- $1 \alpha$ to $\beta$-actin was used to explain the intensity of the sample expression.

\section{Statistical analysis}

Experimental data are reported as means $\pm \mathrm{SD}$. The Student $t$-test and analysis of variance (ANOVA) were performed using SPSS13.0. $\mathrm{P}<0.05$ was considered to be statistically significant. 


\section{RESULTS}

\section{Screening of the effective shRNA}

In the electrophoretic displays of the RT-PCR amplification product, specific VEGF gene strips were observed at $280 \mathrm{bp}$ for each group; $\beta$-actin strips were at $98 \mathrm{bp}$. The strips were analyzed using the gel imaging system, and the average expression levels of VEGF mRNA were as follows: $\mathrm{pVEGF} 1$ group $=0.23 \pm 0.1 ; \mathrm{pVEGF} 2$ group $=0.48 \pm 0.2$; negative plasmid $\mathrm{pNeg}$ group $=0.79 \pm 0.1$; untransfected group $=0.88 \pm 0.2$. The expression of the VEGF mRNA in the negative plasmid pNeg group was not significantly different from that of the untransfected group $(\mathrm{P}>0.05)$, whereas the other two groups ( $\mathrm{pVEGF} 1$ and $\mathrm{pVEGF} 2)$ showed decreased expression to varying degrees $(\mathrm{P}<0.05)$. The $\mathrm{pVEGF} 1$ group exhibited the highest inhibition rate $(82.17 \pm 4.45 \%)$, which was then used for subsequent experiments (Figure 1).

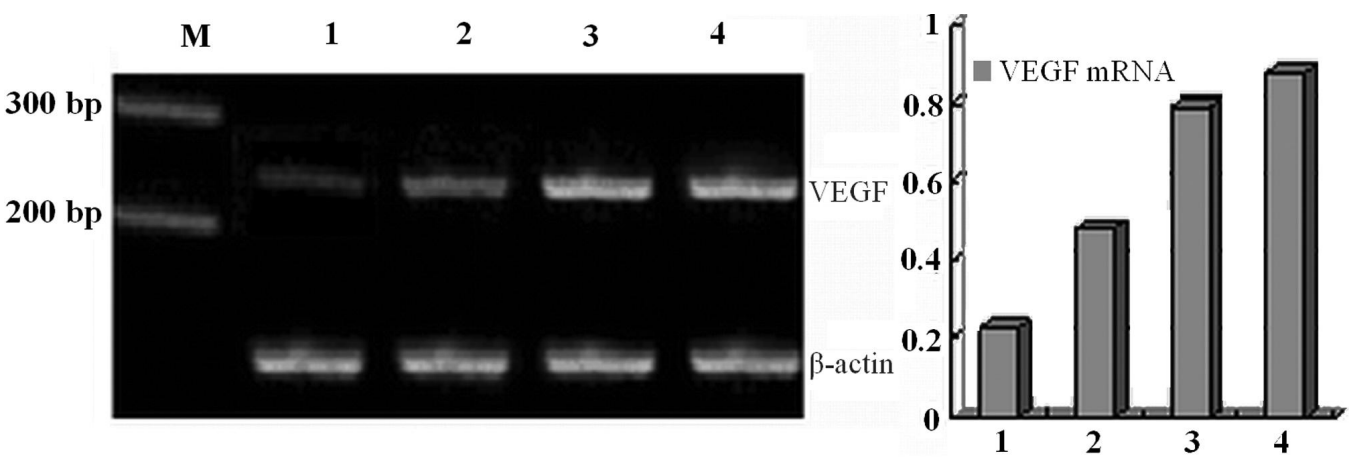

Figure 1. Experssion of VEGF mRNA in transfected SiHa cells (48 h). Lane $M=$ Marker; lane $1=$ pVEGF1 group; lane 2 = pVEGF2 group; lane 3 = pNeg group; lane $4=$ untransfected group.

\section{Cell apoptosis}

The flow cytometry results showed that the apoptosis rates in the pVEGF1 group, pNeg group, and untransfected group were $47.85 \pm 4.65 \%, 9.76 \pm 2.12 \%$, and $6.36 \pm 3.74 \%$, respectively, in which the $\mathrm{pNeg}$ and untransfected groups had no significant difference ( $\mathrm{P}$ $>0.05)$. However, compared with the other two groups, the apoptosis rate of the pVEGF1 group increased significantly $(\mathrm{P}<0.05)$. Thus, VEGF shRNA downregulated the expression of VEGF mRNA while promoting the apoptosis of SiHa cells, as shown in Figure 2.

\section{Growth inhibitory effect}

Nude mice were kept under normal mental status and were given free access to diet and water. No deaths occurred and no significant systemic adverse reactions or body mass changes were observed. The volume of the tumors showed no obvious interclass differences. The tumor growth speed of the blank control group was the fastest, whereas those of the other groups were reduced to some extent (Figure 3). The VEGF shRNA group showed little growth indication within the 6 days before treatment, after which slow growth began to appear, but the 
rate was still lower than that of the control group. The radiotherapy group showed no growth trends between the 6th and 15th days after treatment. The combined therapy group showed the same growth trend as that of the VEGF shRNA group; however, the tumors were reduced in size. Except for the VEGF shRNA group $(\mathrm{P}>0.05)$, the difference in tumor volume between the radiotherapy group, combined treatment group, and blank control group were significant $(\mathrm{P}<0.01)$ at the 12 th day of treatment. The tumor growth inhibitory rates of the VEGF shRNA group, the radiotherapy group, and the combined treatment group were $29.05 \%, 64.38 \%$, and $76.35 \%$, respectively, suggesting that the combined treatment had the strongest inhibitory role, even at the earlier stage.
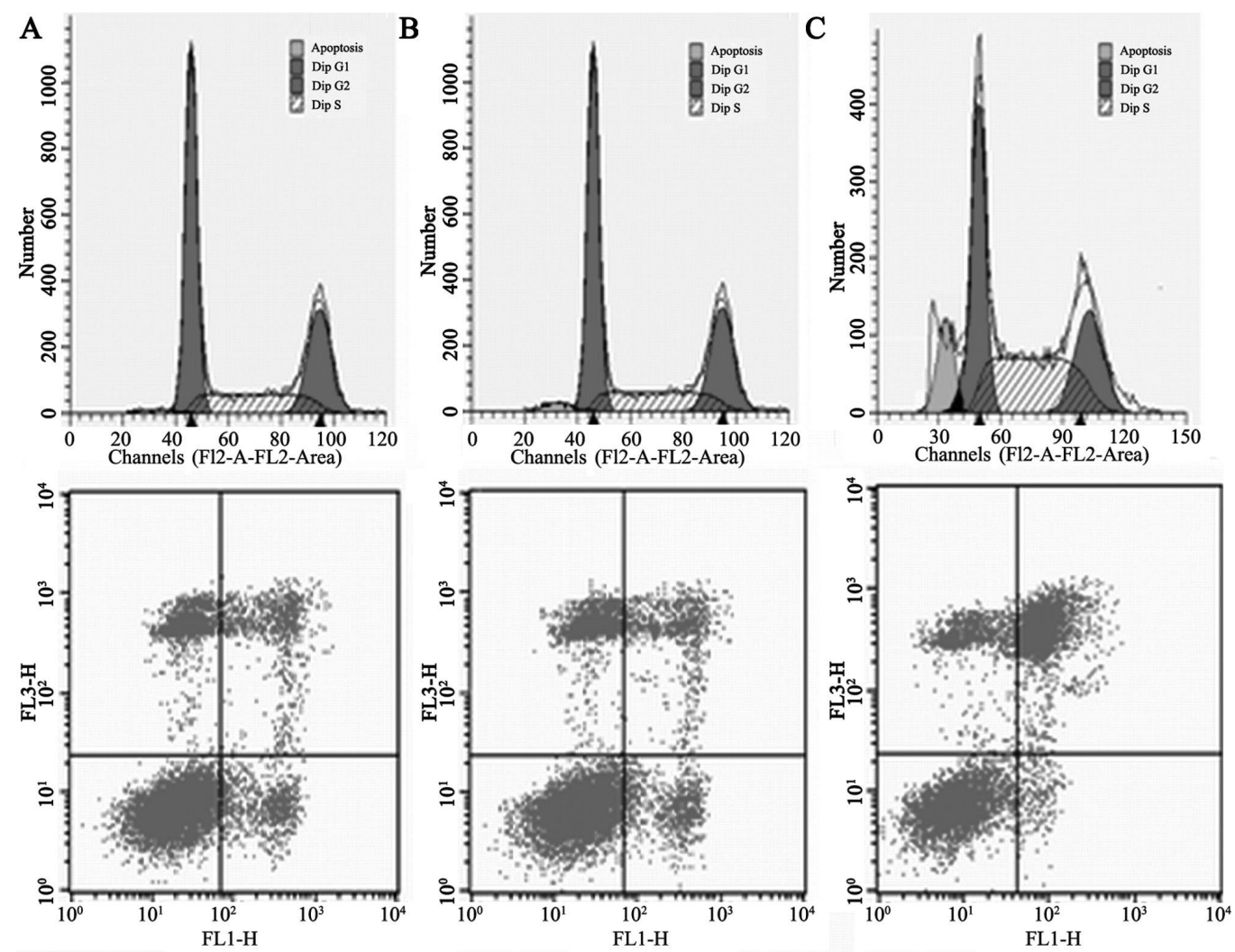

Figure 2. Influence on apoptosis of transfected SiHa cells (48h). A. Untransfected group; B. pNeg group; C. pVEGF1 group.

\section{Histopathological changes}

The histopathological changes of the xenograft tumor under optical microscope showed that tumor cells were abundant in the blank control group and obvious atypia was observed in the nest. In the other three treatment groups, the number of tumor cells decreased, cellular atypia were not obvious, and fibrous tissue hyperplasia and lymphocyte infiltration were significantly reduced compared to the control group. A large number of necrotic and 
apoptotic cells were visible in the radiotherapy group and the combined therapy group; reduction of tumor cells, fibrous tissue proliferation, and tumor cell necrosis and apoptosis were particularly obvious in the combined therapy group (Figure 4).

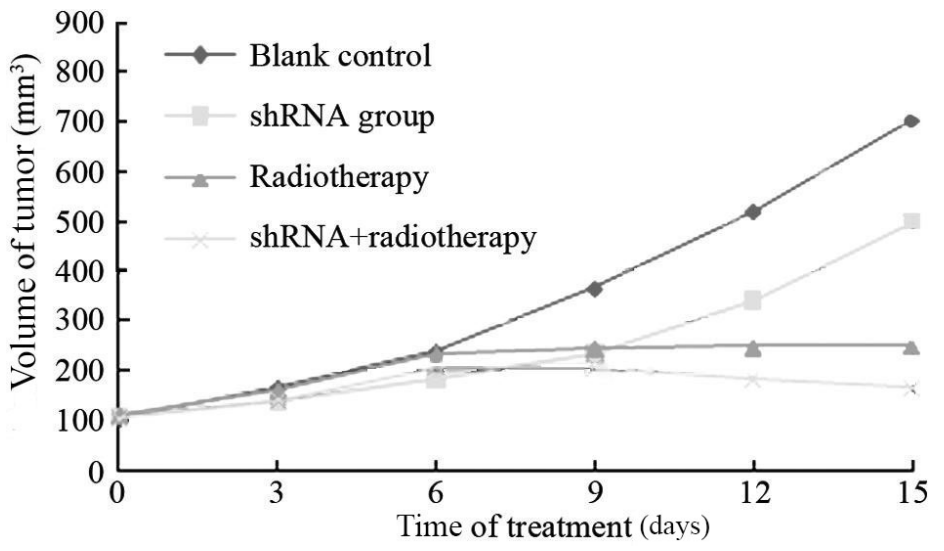

Figure 3. Tumor growth curve of nude mice.

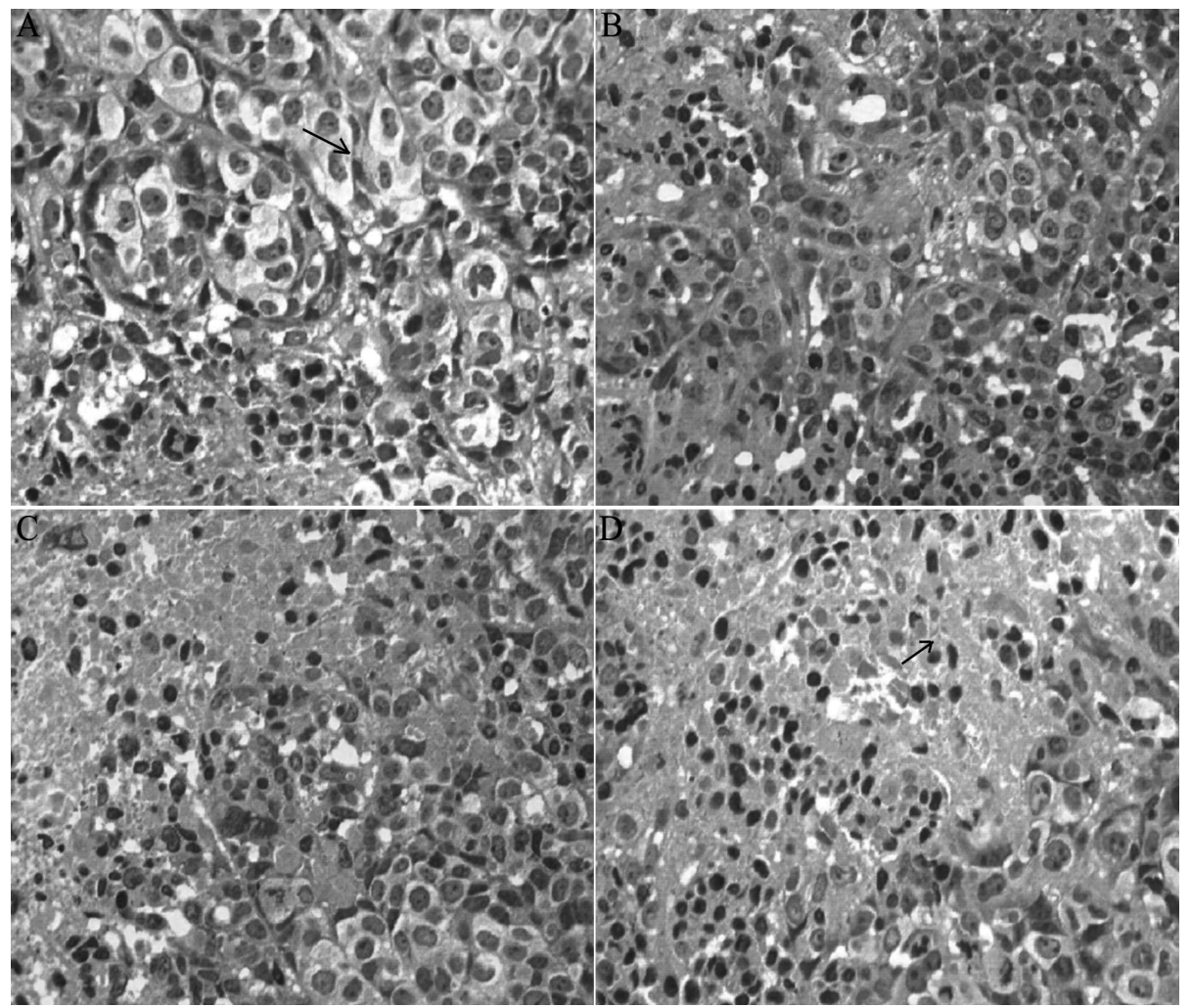

Figure 4. Histopathologic changes under optial microscope (400X). A. Blank control; B. shRNA; C. radiotherapy; D. shRNA + radiotherapy. 


\section{Expression of VEGF protein in transplanted tumor tissues}

The expression of VEGF protein was brownish-yellow in the cytoplasm. The blank control group exhibited 31 to $50 \%$ positive cells $(+)$, whereas the corresponding VEGF protein in the radiotherapy group had a significantly higher proportion $(\mathrm{P}<0.05)$ of positive cells that was $>50 \%(+++)$. In the shRNA group, the proportion of positive VEGF protein cells decreased $(\mathrm{P}<0.01)$ to $<10 \%(-)$, and that in the combined treatment group also decreased $(\mathrm{P}<$ $0.05)$ to $10-30 \%(+)$ (Figure 5). These results suggested that VEGF shRNA interference could downregulate the expression of the VEGF protein in the SiHa cells, and the expression of the VEGF protein induced by radiotherapy was also downregulated.

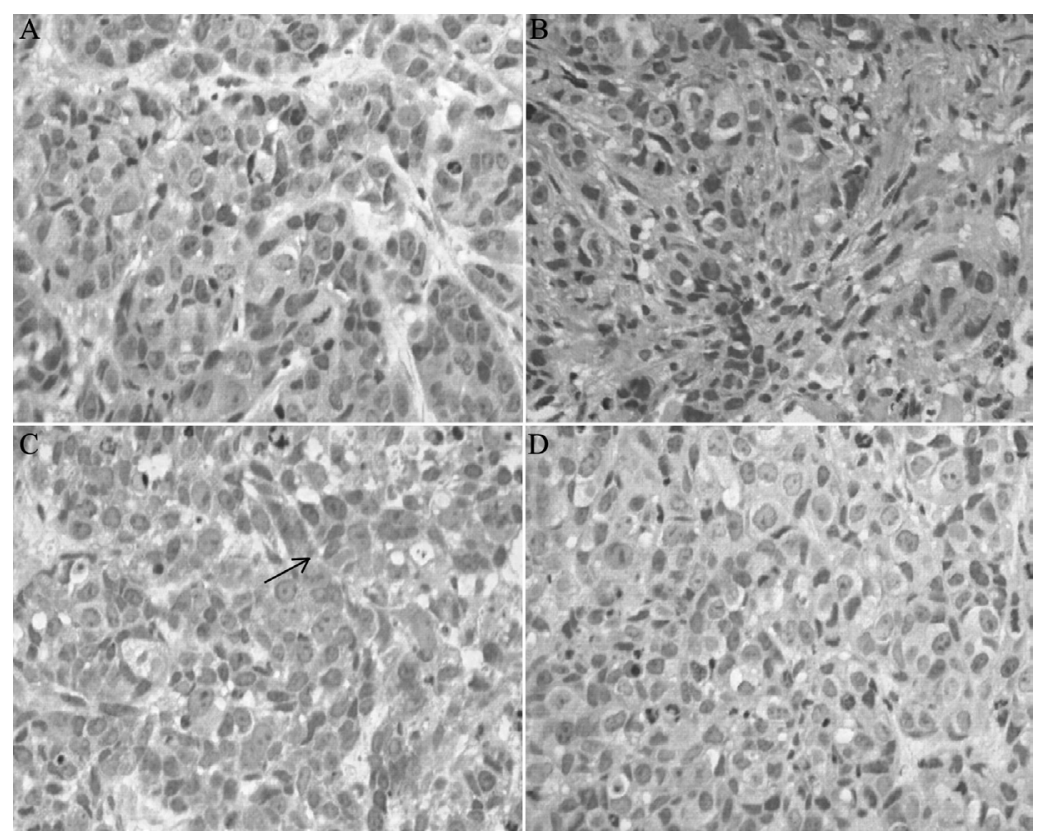

Figure 5. Expression of VEGF protein under optial microscope (400X). A. Blank control; B. shRNA; C. radiotherapy; D. shRNA + radiotherapy.

\section{MVD of xenograft tumors}

The angiogenesis of the radiotherapy group was obvious, and the vascular basement membrane was incomplete. Its MVD was $56.2 \pm 1.4$, which was much higher than that of the control group $(42.9 \pm 1.2)(\mathrm{P}<0.05)$. Angiogenesis decreased in the shRNA $(16.5 \pm 2.3)$ and combined treatment group $(20.5 \pm 0.5)$, whereas the vascular basement membrane was complete. The MVD was much lower than that of the control group $(\mathrm{P}<0.05)$, and the MVD of the combined treatment group was $20.5 \pm 0.5$, which was significantly lower than that of the radiotherapy group $(\mathrm{P}<0.05)$. Radiotherapy could therefore induce tumor angiogenesis and neovascular structural abnormalities. VEGF shRNA could inhibit tumor angiogenesis effectively; this inhibition was maintained in radiation-induced angiogenesis (Table 3). 
Table 3. Microvascular density (MVD) of xenograft tumor.

\begin{tabular}{lcc}
\hline Group & MVD (means \pm SD) & P value (between two groups) \\
\hline Blank control & $42.9 \pm 1.2$ & \\
shRNA & $16.5 \pm 2.3$ & $\mathrm{P}<0.05$ \\
therapy & $56.2 \pm 1.4$ & $\mathrm{P}<0.05$ \\
shRNA+therapy & $20.5 \pm 0.5$ & $\mathrm{P}<0.01$ \\
\hline
\end{tabular}

\section{Expression of HIF-1 $\alpha$ and VEGF proteins in xenograft tumor tissues}

Compared with the blank control group, VEGF shRNA interference could inhibit VEGF and HIF- $1 \alpha$ proteins $(\mathrm{P}<0.05)$ in transplanted tumor tissues and had the same effects on radiation-induced VEGF and HIF-1 $\alpha \alpha$ proteins $(\mathrm{P}<0.05)$ (Figure 6$)$. The hypoxia of the xenograft tumor tissues and the elevated expression of HIF-1 $\alpha \alpha$ may have been aggravated by the radiotherapy, whereas the expression of the VEGF protein in xenograft tumor tissues was inhibited by VEGF shRNA. The vascular functions and hypoxia were improved, whereas the expression of HIF-1 $\alpha$ was decreased.

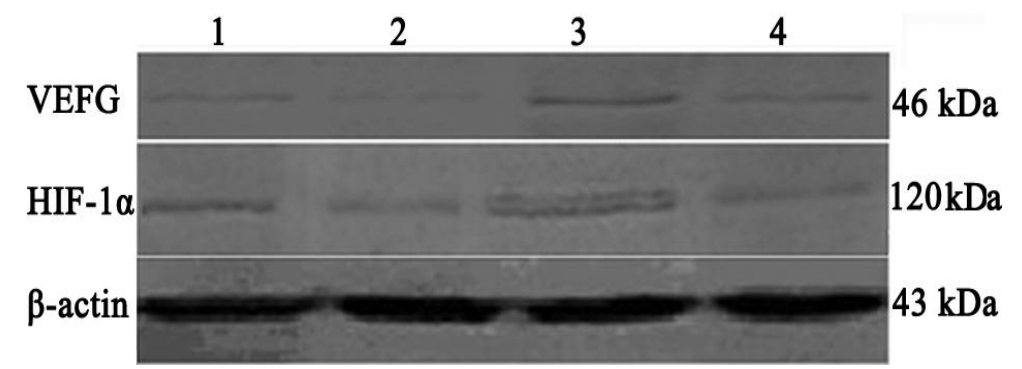

Figure 6. Expression of HIF-1 $\alpha$, VEGF protein in xenograft tumor tissue. Lane $1=$ blank control; lane 2 = shRNA; lane $3=$ therapy; lane $4=$ shRNA + therapy.

\section{DISCUSSION}

Hypoxia can induce angiogenesis, which is the basis of tumor growth and metastasis, and is also an important factor contributing to poor prognosis. In 1971, Dr. Folkman of Harvard University proposed a tumor angiogenesis-dependent doctrine, which stated that the development of tumors was not only related to the potential malignant cells of high proliferation, but also required the support of tumor peripheral vasculature (Folkman, 1971). The study found that the volume of a tumor is usually $<2 \mathrm{~mm}^{3}$ if there is an inadequate blood supply. This value is the maximum size that would ensure nutrient diffusion from the blood vessels to the surrounding tissue, and further proliferation would require neovascularization. Therefore, angiogenesis and growth of the tumor have received wide attention in cancer therapy.

The mechanism of angiogenesis is complex, and VEGF is considered to be one of the most important promoters of angiogenesis (Fujimoto et al., 1998). This study found that the hypoxic state and abnormal microenvironment could improve the expression of VEGF. VEGF, by binding with its receptor, acts on vascular endothelial cells and tumor cells in paracrine and autocrine manners, and then influences the proliferation of vascular endothelial cells, basal 
membrane hydrolysis, the formation of blood vessels, and the biological behavior of tumor cells (Bremnes et al., 2006). The synthesis and secretion of VEGF (Hammes et al., 2008; Samouelian et al., 2008) increases in the normal cervical epithelium, inducing cervical epithelium damage, gradually increasing the number of cancerous cells. In addition, its angiogenic capacity increases significantly along with the elevated expression of VEGF. VEGF is a positive regulator in cervical cancer angiogenesis, and thus plays an important regulatory role in cervical cancer angiogenesis. In cervical cancer, the inhibition of VEGF and its receptor signaling pathway can induce apoptosis of tumor cells, suppression of tumor angiogenesis, and inhibition of tumor growth.

We interfered with the expression of VEGF in SiHa cells via RNAi technology. The double-stranded RNA was processed into 21-25 nucleotides small interference RNA (siRNA) by an endonuclease III-like protein. SiRNA, helicase, and nucleic acid enzymes bound to the RNA-induced silencing complex (RISC), and the RISC cleaved the homologous target mRNA specifically guided by siRNA, which resulted in deletion of the gene after degradation of the target mRNA(Allen et al., 2007). Compared with antisense RNA, ribozymes, gene knockout, and gene containment, this method is more efficient, more specific, and more convenient. The interference sequence with the stem-loop structure was inserted into the eukaryotic promoters with U6 or H1. Afterward, the shRNA vector was constructed, the hairpin parts of the shRNA were cut using the Dicer enzyme, and the siRNA was formed (Vermeulen et al., 2005). These shRNA vectors are more stable, efficient, and last longer compared to the direct synthesis of siRNA. We screened the efficiency of the VEGF shRNA sequence to inhibit the expression of cellular endogenous VEGF mRNA in vitro. $\mathrm{SiHa}$ cell apoptosis was induced, whereas the expression of VEGF protein and angiogenesis in the transplanted tumor tissue was downregulated. Finally, the tumor growth was inhibited. However, we also found that this inhibitory effect was temporary, and the growth of the xenograft tumor was restored without the intervention of VEGF shRNA.

A growing number of studies have found that the levels of VEGF have increased after radiotherapy in a variety of tumor tissues (Hovinga et al., 2005). Hypoxia has been regarded as the most important factor in this event (Patt et al., 1998). HIF-1 $\alpha$ is an indirect marker of cell hypoxia and is the upstream regulatory gene of VEGF. Gorski et al. (1999) suggested that radiotherapy plays a dual role for VEGF. On the one hand, the reduction of tumor burden after radiotherapy shows a declining trend of VEGF. On the other hand, tumor vascular damage induced by radiotherapy worsens the hypoxia compared to the induced expression of VEGF. In the radiotherapy group, all nude mice showed inhibition of HIF-1 $\alpha$, and the expression of VEGF increased in tumor tissues, which is consistent with previous reports. A protective mechanism can be induced in tumors through HIF-1 $\alpha$-VEGF neovascularization. Hypoxia is caused by the structural and functional abnormalities of new tumor blood vessels (Jain, 2001). This status will increase the resistance of tumors to chemoradiotherapy, and will also elevate its invasiveness.

Studies have shown that radiotherapy and anti-angiogenesis therapy have very good synergy. Jain (2005) suggested that angiogenesis inhibitors could normalize the structural and functional tumor abnormality before the vasculature subsided, thereby improving the tumor microenvironment and reducing tumor hypoxia. The angiogenesis inhibitors reduce the tumor MVD while improving the blood supply to alleviate tumor hypoxia. This inconsistent change is achieved by the elimination of immature tumor vessels and the reduction in the number of aerobic cells and vascular permeability. Luo et al. (2005) investigated the influence of mouse endostatin combined with radiotherapy in a lung cancer model by using a pXLG-mEndo plasmid. The addition of pXLG-mEndo increased the levels of endostatin in the tumor tissue, and 
the growth of tumors in mice was significantly suppressed. Shibuya et al. (2007) also showed that ZD6474 (an anti-angiogenic drug) could reduce the expression levels of VEGF and epidermal growth factor receptor (EGFR) in tumor tissues, thereby increasing the sensitivity of non-small cell lung cancer for radiotherapy. Recent fundamental research further confirmed that anti-angiogenesis in combined therapy could increase the anti-tumor effect of radiotherapy in human nasopharyngeal cancer and lung adenocancer (Wen et al., 2009).

Anti-VEGF treatment can block the protection of VEGF for the radiation-induced apoptosis of vascular endothelial cells. Combined with radiotherapy, it can reduce newborn tortuous vessel cavities and osmotic pressure, resulting in the reperfusion of a part of the compressive deformation invalid vessel, remodeling of the vascular system, improvement of the serious hypoxic state of the tumor, and improvement of the radiation sensitivity of the tumor. This constitutes the theoretical basis of the combination therapy of radiation and anti-VEGF. Winkler et al. (2004) found that anti-angiogenic therapy could induce the emergence of a "vascular normalization window period" in tumor blood vessels. This phenomenon usually appears between the 4th and the 6th days after anti-angiogenic therapy. In this window period, the functions of tumor blood vessels and normal vessels are similar, the level of oxygen in tumors is high, and tumor cells are sensitive to radiation. Thus, radiotherapy has better synergistic anti-tumor effects in the window period. Based on the above considerations, we carried out anti-angiogenic therapy using VEGF shRNA at the 5th day after radiotherapy. Radiotherapy combined with VEGF shRNA anti-angiogenic therapy not only inhibited the growth of tumors, but also induced tumor regression. Thus, the results were in accordance with what was expected: the growth inhibition rate of tumors was $76.35 \%$. The immunohistochemistry pathological analysis on transplanted tumor tissues showed that radiotherapy combined with VEGF shRNA anti-angiogenic therapy reduced the tumor cells and cell atypia significantly. A large number of interstitial tissues were replaced, and apoptosis was increased. Compared with radiotherapy alone, the combined treatment effectively inhibited increased MVD and HIF-1 $\alpha$ after radiotherapy, improved the hypoxic state of the tumor microenvironment, enhanced the killing effect of the radiation, and increased tumor radiosensitivity. In summary, combined therapy could overcome the limitations of individual treatment, had no significant adverse reactions, and achieved the strongest inhibitory effect.

In summary, VEGF shRNA antiangiogenic therapy combined with radiotherapy could inhibit the growth of cervical cancer SiHa xenografts in nude mice, increase the radiosensitivity of cervical cancer, and achieve a synergistic antitumor effect. The "vascular normalization" effects of angiogenesis inhibitors are temporary and some researchers have found that it may be reversible (Batchelor et al., 2007); thus, the beginning and ending time are vital for a rational combined treatment program. Choosing a suitable time to combine radiotherapy with the angiogenesis inhibitors to obtain the best inhibitory effect will be the focus of further studies about combination therapy. Eschenbach, the chairman of the U.S. National Cancer Institute, pointed out that cancer treatment was a "seek and destroy" strategy in the 20th century, whereas in the 21 st century, it has become a "targeting and control" strategy. With the development of molecular oncology, research about the combined therapies and clinical applications of vascular targeting drugs have exciting prospects for the treatment of cervical cancer and other solid tumors.

\section{REFERENCES}

Allen D, Kenna PF, Palfi A, McMahon HP, et al. (2007). Development of strategies for conditional RNA interference. $J$. Gene. Med. 9: 287-298. 
Batchelor TT, Sorensen AG, di Tomaso E, Zhang WT, et al. (2007). AZD2171, a pan-VEGF receptor tyrosine kinase inhibitor, normalizes tumor vasculature and alleviates edema in glioblastoma patients. Cancer Cell 11: 83-95.

Bremnes RM, Camps C and Sirera R (2006). Angiogenesis in non-small cell lung cancer: the prognostic impact of neoangiogenesis and the cytokines VEGF and bFGF in tumours and blood. Lung Cancer 51: 143-158.

Folkman J (1971). Tumor angiogenesis: therapeutic implications. N. Engl. J. Med. 285: 1182-1186.

Fujimoto K, Hosotani R, Wada M, Lee JU, et al. (1998). Expression of two angiogenic factors, vascular endothelial growth factor and platelet-derived endothelial cell growth factor in human pancreatic cancer, and its relationship to angiogenesis. Eur. J. Cancer 34: 1439-1447.

Gorski DH, Beckett MA, Jaskowiak NT, Calvin DP, et al. (1999). Blockage of the vascular endothelial growth factor stress response increases the antitumor effects of ionizing radiation. Cancer Res. 59: 3374-3378.

Hammes LS, Tekmal RR, Naud P, Edelweiss MI, et al. (2008). Upregulation of VEGF, c-fms and COX-2 expression correlates with severity of cervical cancer precursor (CIN) lesions and invasive disease. Gynecol. Oncol. 110: 445451.

Hovinga KE, Stalpers LJ, van Bree C, Donker M, et al. (2005). Radiation-enhanced vascular endothelial growth factor (VEGF) secretion in glioblastoma multiforme cell lines - a clue to radioresistance? J. Neurooncol. 74: 99-103.

Jain RK (2001). Normalizing tumor vasculature with anti-angiogenic therapy: a new paradigm for combination therapy. Nat. Med. 7: 987-989.

Jain RK (2005). Normalization of tumor vasculature: an emerging concept in antiangiogenic therapy. Science 307: 58-62.

Kamangar F, Dores GM and Anderson WF (2006). Patterns of cancer incidence, mortality, and prevalence across five continents: defining priorities to reduce cancer disparities in different geographic regions of the world. J. Clin. Oncol. 24: $2137-2150$

Luo X, Slater JM and Gridley DS (2005). Enhancement of radiation effects by pXLG-mEndo in a lung carcinoma model. Int. J. Radiat. Oncol. Biol. Phys. 63: 553-564.

McDermott DF and George DJ (2010). Bevacizumab as a treatment option in advanced renal cell carcinoma: an analysis and interpretation of clinical trial data. Cancer Treat. Rev. 36: 216-223.

Miller K, Wang M, Gralow J, Dickler M, et al. (2007). Paclitaxel plus bevacizumab versus paclitaxel alone for metastatic breast cancer. N. Engl. J. Med. 357: 2666-2676.

O'Reilly MS (2006). Radiation combined with antiangiogenic and antivascular agents. Semin. Radiat. Oncol. 16: 45-50.

Parkin DM, Pisani P and Ferlay J (1999). Global cancer statistics. CA Cancer J. Clin. 49: 33-64, 1.

Patt S, Danner S, Theallier-Janko A, Breier G, et al. (1998). Upregulation of vascular endothelial growth factor in severe chronic brain hypoxia of the rat. Neurosci. Lett. 252: 199-202.

Samouelian V, Revillion F, Alloy N, Lhotellier V, et al. (2008). Measurement of mRNA of 11 biomarkers by RT-PCR to detect lymph node involvement in cervical cancer. Int. J. Biol. Markers 23: 74-82.

Shibuya K, Komaki R, Shintani T, Itasaka S, et al. (2007). Targeted therapy against VEGFR and EGFR with ZD6474 enhances the therapeutic efficacy of irradiation in an orthotopic model of human non-small-cell lung cancer. Int. J. Radiat. Oncol. Biol. Phys. 69: 1534-1543.

Vermeulen A, Behlen L, Reynolds A, Wolfson A, et al. (2005). The contributions of dsRNA structure to Dicer specificity and efficiency. RNA 11: 674-682.

Wen QL, Meng MB, Yang B, Tu LL, et al. (2009). Endostar, a recombined humanized endostatin, enhances the radioresponse for human nasopharyngeal carcinoma and human lung adenocarcinoma xenografts in mice. Cancer Sci. 100: 1510-1519.

Winkler F, Kozin SV, Tong RT, Chae SS, et al. (2004). Kinetics of vascular normalization by VEGFR2 blockade governs brain tumor response to radiation: role of oxygenation, angiopoietin-1, and matrix metalloproteinases. Cancer Cell 6: 553-563. 